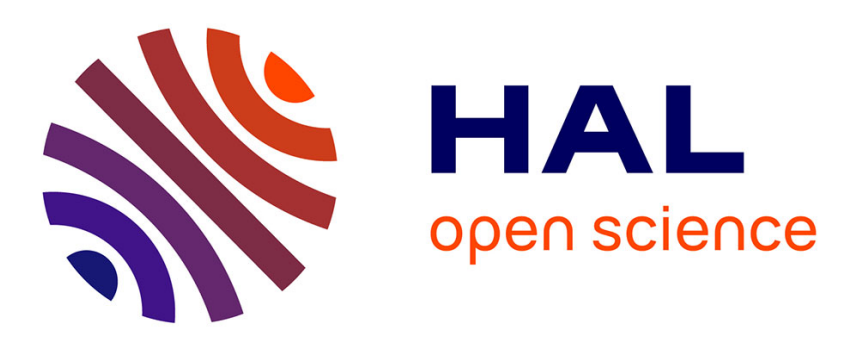

\title{
Kite Generator System Periodic Motion Planning Via Virtual Constraints
}

\author{
M. Ahmed, Ahmad Hably, Seddik Bacha
}

\section{To cite this version:}

M. Ahmed, Ahmad Hably, Seddik Bacha. Kite Generator System Periodic Motion Planning Via Virtual Constraints. IECON 2013 - 39th Annual Conference of the IEEE Industrial Electronics Society, Nov 2013, Vienne, Austria. pp.1-6. hal-00873092

\section{HAL Id: hal-00873092 \\ https://hal.science/hal-00873092}

Submitted on 15 Oct 2013

HAL is a multi-disciplinary open access archive for the deposit and dissemination of scientific research documents, whether they are published or not. The documents may come from teaching and research institutions in France or abroad, or from public or private research centers.
L'archive ouverte pluridisciplinaire HAL, est destinée au dépôt et à la diffusion de documents scientifiques de niveau recherche, publiés ou non, émanant des établissements d'enseignement et de recherche français ou étrangers, des laboratoires publics ou privés. 


\section{Kite Generator System Periodic Motion Planning Via Virtual Constraints}

\author{
Mariam Ahmed ${ }^{1}$ \\ Grenoble Electrical Engineering \\ Laboratory (G2ELab) \\ 38402 Saint-Martin d'Heres,
}

France

\author{
Ahmad Hably ${ }^{2}$ \\ GIPSA-lab -ENSE3 BP 46 \\ 38402 Saint-Martin d'Heres, \\ France
}

\author{
Seddik Bacha ${ }^{3}$ \\ Grenoble Electrical Engineering \\ Laboratory (G2ELab) \\ 38402 Saint-Martin d'Heres, \\ France
}

\begin{abstract}
This paper presents a new control strategy for Kite Generator System (KGS). The proposed feedback strategy is based on motion planning using the virtual constraint approach and ensures exponential orbital stability of the desired trajectory. The strategy is detailed, applied and tested via numerical simulations and showed good convergence to a desired periodic motion.
\end{abstract}

\section{INTRODUCTION}

The so-called kite generator system (KGS) is a kitebased wind energy generator. A mechanical power is generated when one or several tethered airfoils (normally light weighted kites) are pulled by wind. This power is then transformed into an electrical energy by means of an onground generator.

In general, three types of kite-based systems were proposed: Pumping-type [4][7], closed-loop [1][3], and carousel systems [12]. These new concepts and are currently heavily investigated by many research groups and start-up companies. Fig. 1 shows the main projects mounted in Western Europe (for more details, see the recent survey written by the authors [2]). Kite-based systems are expected to produce huge amounts of power using a simple and safe structure, but they are challenged by a complexity of their modeling and control, since they are nonlinear, constrained, and highly unstable in the open-loop. As well, due to limitation of the tether's length and power region, the kite should always be redrawn to its initial position to start a new cycle, a phase consumes energy. This traction-recovery functioning yields a generating-consumption cycle that needs to be optimized. This is achieved by controlling the kite to follow a certain trajectory via control of the kite's attack and/or roll angle, and the tether's traction that is controlled via the ground generator torque [2]. The trajectory choice is done through a maximization of the produced power, and the kite is controlled to track this trajectory through an orientation mechanism. Control methods vary from simple linear control [19], observer-based control [10], to nonlinear model predictive control (NMPC) [11], [1] and neural network control [9]. In this paper, a virtual constraint based control for orbit stabilization of kite's trajectories is proposed. Applying this

\footnotetext{
${ }^{1}$ Mariam. AHMED@g2elab.grenoble-inp. fr

${ }^{2}$ Corresponding author

Ahmad.Hablyagrenoble-inp.fr

${ }^{3}$ Seddik. Bachalg2elab.grenoble-inp.fr
}

method offers a simple yet efficient control, that generates an exponentially orbitally stable trajectory for the KGS. As a first step in this application, the aim is to work with an increasing level of complexity, starting from a relatively simple kite-based system design, whose structure is shown in Fig.2. Note that all of the principles explained in this paper can be used as a basis for more complex systems.

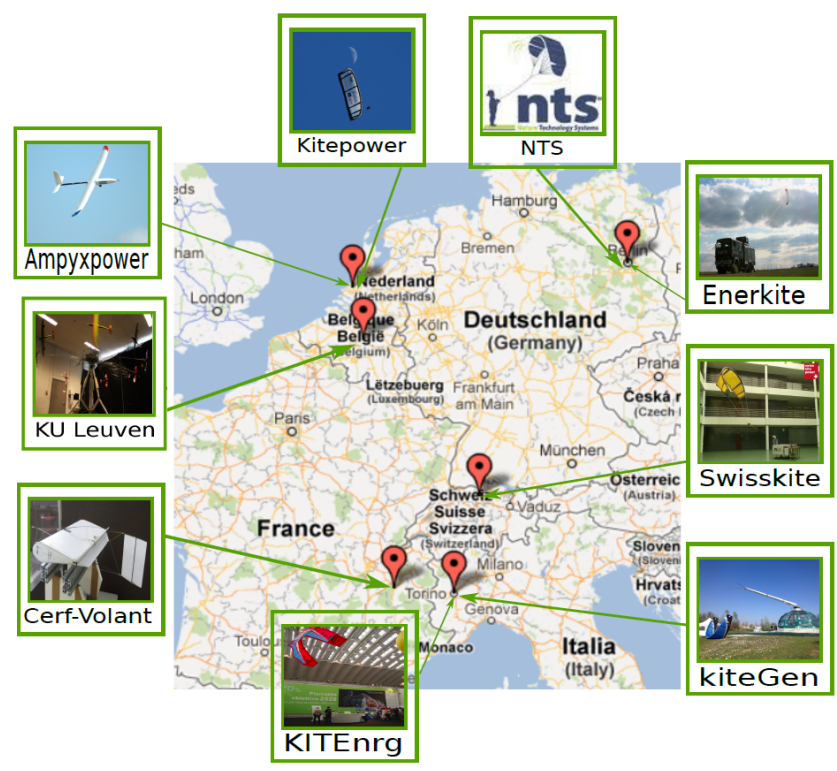

Fig. 1. Overview of the main existing European research groups exploring the wind kites principle.

The paper is structured as follows. In section II, a brief recall of virtual constraints control methods is given. A description of the KGS dynamic model and its virtual constraint reduced model are presented in section III. The proposed control law is designed in section IV followed by simulation results in section $\mathrm{V}$. The paper ends with conclusions and perspectives (section VI).

\section{Virtual Constraint Approaches}

Virtual constraints (VC) has emerged recently as a valuable tool to solve motion control problems. For an underactuated Euler-Lagrange system, VC are defined as relations among the system's variables and are enforced by feedback, which leads to reducing the system's degrees of freedom. 


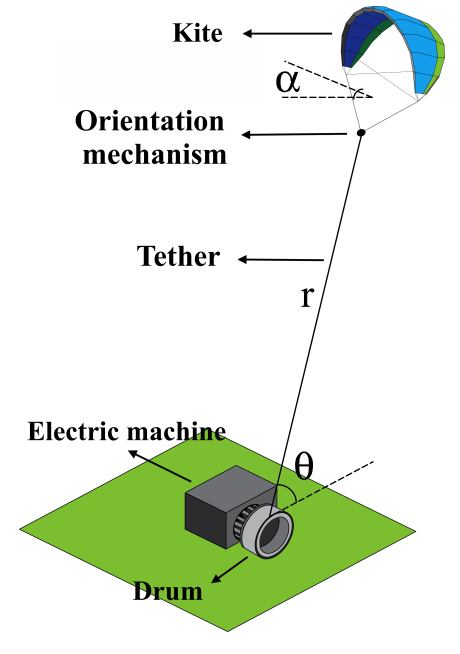

Fig. 2. A simplified Kite Generator System with $r$ length of the tether, $\theta$ flight angle and $\alpha$ kite's angle of attack.

The goal of the feedback design is either to render an existing periodic motion orbitally stable or to force the system dynamics to generate a new periodic motion and ensure its orbital stability [5][13].

The notion of virtual constraints has been useful to design controllers for biped robots, as well as, control of underactuated 3DOF helicopter movement [18], pendubot [8], and cart-pendulum system [15].

This VC approach seems to be suitable to the control and periodic motion stabilization of the kite generator system, which, to the authors' knowledge, had never been used before for this type of systems. In this paper, the constructive tool for orbital stabilization of under-actuated nonlinear systems proposed by [15] will be applied. It can be summarized by the following steps:

- Finding the Euler-Lagrange model of the system.

- Application of a partial feedback linearization, where the remaining nonlinear part is integrable.

- Construction of an auxiliary linear periodic control system of reduced order.

- Design of a LQR-based control for the auxiliary system.

- A modification of the control developed in the previous item to be applied to the original nonlinear system.

These steps will be developed in details in the following sections with a special attention to the studied kite generator system.

\section{Kite Generator System Dynamic Model}

The considered KGS is shown in Fig.2. Simply stated, the system consists of a tethered kite connected to a generator through a drum. The kite's roll and attack angles are controlled via an orientation mechanism, while the traction exercised on the kite can be controlled by the generator's torque.

The system model is validated in [10], where a pumping mode with a fixed inclination of the tether is adapted and insured using an observer-based control.

\section{A. KGS Under-actuated Model}

The previous system has two degrees of freedom, a translation along the tether $r$, and a rotation $\theta$. The objective is to control both $r$, and $\theta$ by only one input that is the attack angle $\alpha^{1}$. Thus the considered system is an under-actuated system.

To simplify the presentation of the system's model, the following notations are used:

$$
\begin{aligned}
a & =\frac{1}{2} \rho_{a} S \\
b & =a\left(\frac{C_{L}^{2}}{\pi \lambda e}+C_{D_{0}}\right)
\end{aligned}
$$

with:

- $\rho_{a}$ is the air density.

- $A$ is the kite surface.

- $\lambda$ is the kite's aspect ratio.

- $e$ is the Oswald effeciency factor.

- $C_{L}, C_{D}$ are the lift and drag coefficients.

As well, the effective wind velocity is defined as the difference between the wind's and the kite's speed. Its norm and angle are expressed in the polar coordinates $(r, \theta)$ in eq. 1 .

$$
\begin{aligned}
& W_{e}^{2}=(r \dot{\theta} \cos \theta+\dot{r} \sin \theta)^{2}+(V+r \dot{\theta} \sin \theta+\dot{r} \cos \theta)^{2} \\
& \alpha_{w}=-\arctan \left(\frac{r \dot{\theta} \cos \theta+\dot{r} \sin \theta}{V+r \dot{\theta} \sin \theta+\dot{r} \cos \theta}\right)
\end{aligned}
$$

with $V$ being the wind speed.

The previously defined notations allow writing the KGS dynamic model by the equations eq. 2 and eq. 3 .

$$
\begin{aligned}
& \left\{\begin{array}{l}
\ddot{\theta}+\frac{2 \dot{r} \dot{\theta}}{r}+\frac{1}{r M}\left(b v_{r}^{2} \sin \left(\theta-\alpha_{w}\right)\right. \\
\left.-a v_{r}^{2}\left(\frac{\partial C_{L}}{\partial \alpha} \alpha_{w}+C_{L 0}\right) \cos \left(\theta-\alpha_{w}\right)+W \cos \theta\right) \\
=\frac{a v_{r}^{2}}{r M} \frac{\partial C_{L}}{\partial \alpha} \cos \left(\theta-\alpha_{w}\right) \alpha_{u}
\end{array}\right. \\
& \left\{\begin{array}{l}
\ddot{r}-\frac{M}{M+M_{I M}} r \dot{\theta}^{2}+\frac{1}{M+M_{I M}}\left(b v_{r}^{2} \cos \left(\theta-\alpha_{w}\right)\right. \\
\left.+a v_{r}^{2}\left(\frac{\partial C_{L}}{\partial \alpha} \alpha_{w}+C_{L 0}\right) \sin \left(\theta-\alpha_{w}\right)-W \cos \theta-T\right) \\
=\frac{a v_{r}^{2}}{M+M_{I M}} \frac{\partial C_{L}}{\partial \alpha} \sin \left(\theta-\alpha_{w}\right) \alpha_{u}
\end{array}\right.
\end{aligned}
$$

with:

- $M$ is the system's flying part mass

- $M_{I M}$ is the rotor's mass.

- $W$ is the system's flying part weight.

- $\alpha_{u}$ the attack angle control

- $T$ is the tension in the tether.

The KGS dynamics can be equally expressed in the EulerLagrange representation by eq. 4 .

$$
D(\theta, r)\left[\begin{array}{l}
\ddot{\theta} \\
\ddot{r}
\end{array}\right]+C(\theta, r, \dot{\theta}, \dot{r})\left[\begin{array}{l}
\dot{\theta} \\
\dot{r}
\end{array}\right]+\nabla P(\theta, r)=B(\theta, r) \alpha_{u}
$$

where:

- $D(\theta, r)$ is the inertia matrix.

- $C(\theta, r, \dot{\theta}, \dot{r})$ is the Coriolis matrix.

- $P(\theta, r)$ is the potential energy function.

- $B(\theta, r)$ is the control matrix.

The detailed expressions of the previous matrices are given in the Appendix.

\footnotetext{
${ }^{1}$ The tension in the tether is constant in this case.
} 
As noticed, the system can be represented by one equation with two variables and no input. Forcing a virtual constraint to link both variables, leads to a reduced dynamics with one degree of freedom.

\section{B. The Reduced Dynamics system}

As mentioned above, the objective of the VC approach is to find a reduced dynamics system by applying a partial feedback linearization.

The choice of the virtual constraint is determined by the desired orbit of the system hence its functionality. It should, however, respect a few conditions that will be discussed later on. For a start, the function of eq.5 is chosen as the virtual constraint with $d_{0}$ being a constant.

$$
r=\phi(\theta)=\frac{d_{0}}{\sin \theta}
$$

Note that the function can be derived either by observation of some real motion, by analytic design procedures, or by some numerical research as cited in [18].

Suppose that there exists a control law of the under-actuated system of eq. 4 that makes the constraint presented in (eq.5) invariant, then the overall closed-loop system results in an input-free reduced system that can be written in the form of eq.6.

$$
\alpha(\theta) \ddot{\theta}+\beta(\theta) \dot{\theta}^{2}+\gamma(\theta)=0
$$

with:

$$
\begin{aligned}
\alpha(\theta)= & M \phi(\theta) \sin \left(\theta-\alpha_{w}\right)-\left(M+M_{I M}\right) \phi^{\prime}(\theta) \cos \left(\theta-\alpha_{w}\right) \\
\beta(\theta)= & \left(M \phi(\theta)-\left(M+M_{I M}\right) \phi^{\prime \prime}(\theta)\right) \cos \left(\theta-\alpha_{w}\right) \\
& +2 M \phi^{\prime}(\theta) \sin \left(\theta-\alpha_{w}\right)
\end{aligned}
$$$$
\gamma(\theta)=b v_{r}^{2}-W \sin \left(\alpha_{w}\right)-\cos \left(\theta-\alpha_{w}\right) T
$$

where $\phi^{\prime}($.$) and \phi^{\prime \prime}($.$) are respectively the first and second$ derivatives of the virtual constraint with respect to $\theta$.

The chosen virtual constraint should be regular and stabilizable [6]. In order to be regular it should satisfy the condition ([6]-proposition 3.2)

$$
B^{\perp} . D(\hat{\phi}(\theta)) . \hat{\phi}^{\prime}(\theta) \neq 0
$$

with $\hat{\phi}(\theta)=[\theta, \phi(\theta)]$, which implies that the system's variables should respect the condition.

$-M \phi(\theta) \sin \left(\theta-\alpha_{w}\right)+\left(M+M_{I M}\right) \phi^{\prime}(\theta) \cos \left(\theta-\alpha_{w}\right) \neq 0$

It is necessary as well that the virtual constraint is stabilizable, hence, there exists a smooth feedback $u(\theta, \dot{\theta})$ that enforces it. According to [6] parametric VCs $r=\phi(\theta)$, which is the case of the chosen KGS, are stabilisable.

The resulted reduced system is a periodic Euler-Lagrange system [6] (Fig.3), which means that the same feedback strategy, used to enforce the $\mathrm{VC}$, results in generating a periodic motion for all the system's degrees of freedom [17].

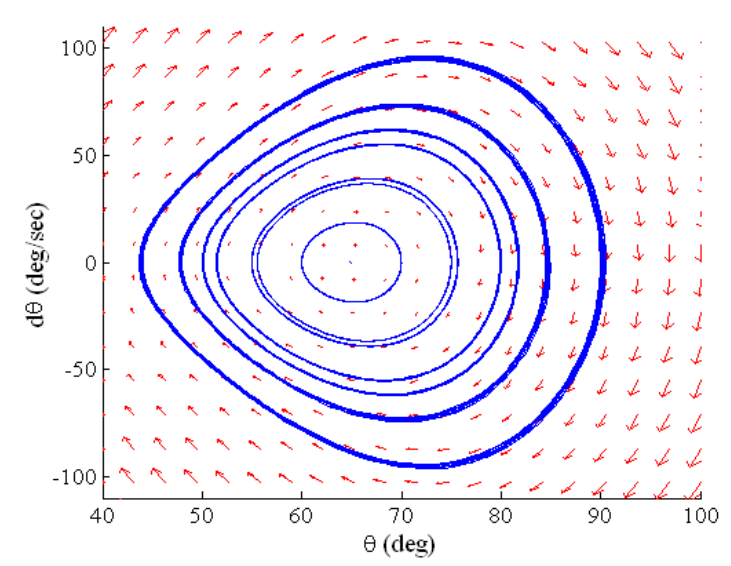

Fig. 3. The reduced system periodic orbits.

The objective is to design the feedback controller that guarantees the invariance of the chosen virtual constraints and an orbital asymptotic stability of the chosen periodic solution. This control problem can be expressed by eq.7

$$
y=r-\phi(\theta)=0, \theta(t)=\theta(t+T)
$$

\section{Partial feedback linearization}

After introducing the problem in the form eq.7, a partial feedback linearisation is applied in order to simplify the stability analysis for the nonlinear system of eq.6 to stability analysis for an auxiliary linear periodic in time controlled system.

Since

$$
\begin{gathered}
r=y+\phi(\theta) \\
\dot{r}=\dot{y}+\phi^{\prime}(\theta) \dot{\theta} \\
\ddot{r}=\ddot{y}+\phi^{\prime \prime}(\theta) \dot{\theta}^{2}+\phi^{\prime}(\theta) \ddot{\theta}
\end{gathered}
$$

the Euler-lagrange system of eq.4, can be written in the coordinates $(\theta, y)$ in eq.8.

$$
\begin{aligned}
& L(\theta, y)\left[\begin{array}{l}
\ddot{\theta} \\
\ddot{y}
\end{array}\right]+N(\theta, y, \dot{\theta}, \dot{y}) \\
& =\left[B(\theta, r) \alpha_{u}-C(\theta, r, \dot{\theta}, \dot{r})\left[\begin{array}{c}
\dot{\theta} \\
\dot{r}
\end{array}\right]-\nabla P(\theta, r)\right]_{r=y+\phi(\theta)}
\end{aligned}
$$

with:

$$
L(\theta, y)=\left[\begin{array}{cc}
1 & 0 \\
\phi^{\prime}(\theta) & 1
\end{array}\right]
$$

and:

$$
N(\theta, y, \dot{\theta}, \dot{y})=\left[\begin{array}{c}
0 \\
\phi^{\prime \prime}(\theta) \dot{\theta}^{2}
\end{array}\right]
$$

The dynamics of the variable $y$ are given by:

$$
\ddot{y}=K(\theta, y) u+R(\theta, y, \dot{\theta}, \dot{y})
$$




$$
\begin{aligned}
K(\theta, y)= & \frac{\partial C_{L}}{\partial \alpha} \frac{a v_{r}^{2}}{M\left(M+M_{I M}\right)(y+\phi)}\left(-\left(M+M_{I M}\right) \phi^{\prime} \cos \left(\theta-\alpha_{w}\right)+M(y+\phi) \sin \left(\theta-\alpha_{w}\right)\right) \\
R(\theta, y, \dot{\theta}, \dot{y})= & \frac{\phi^{\prime}}{M(y+\phi)}\left(2 M\left(\dot{y}+\phi^{\prime} \dot{\theta}\right) \dot{\theta}+b v_{r}^{2} \sin \left(\theta-\alpha_{w}\right)-a v_{r}^{2}\left(\frac{\partial C_{L}}{\partial \alpha} \alpha_{w}+C_{L 0}\right) \cos \left(\theta-\alpha_{w}\right)+W \cos \theta\right)-\phi^{\prime \prime} \dot{\theta}^{2} \\
& +\frac{1}{M+M_{I M}}\left(M(y+\phi) \dot{\theta}^{2}+b v_{r}^{2} \cos \left(\theta-\alpha_{w}\right)+a v_{r}^{2}\left(\frac{\partial C_{L}}{\partial \alpha} \alpha_{w}+C_{L 0}\right) \sin \left(\theta-\alpha_{w}\right)-W \cos \theta-T\right)
\end{aligned}
$$

where $K(),. R($.$) are given in eq. 10$.

According to eq.9, using the feedback transformation:

$$
u=K^{-1}(y, \theta)[v-R(\theta, y, \dot{\theta}, \dot{y})]
$$

results in a partly linear system:

$\left\{\begin{array}{l}\alpha(\theta) \ddot{\theta}+\beta(\theta) \dot{\theta}^{2}+\gamma(\theta)=g_{y}(\theta, \dot{\theta}, \ddot{\theta}) y+g_{\dot{y}}(\theta, \dot{\theta}) \dot{y}+g_{v}(\theta) \\ \ddot{y}=v\end{array}\right.$

with,

$$
\begin{aligned}
g_{y} & =-M \sin \left(\theta-\alpha_{w}\right) \ddot{\theta}-M \cos \left(\theta-\alpha_{w}\right) \dot{\theta}^{2} \\
g_{\dot{y}} & =2 M \dot{\theta} \sin \left(\theta-\alpha_{w}\right) \\
g_{v} & =\left(M+M_{I M}\right) \cos \left(\theta-\alpha_{w}\right)
\end{aligned}
$$

Let $\left(\theta_{*}, \dot{\theta}_{*}\right)$ be the reference orbit, if $\alpha\left(\theta_{*}(t)\right) \neq 0, \forall t \in$ $\left[0, T_{p}\right]$, then the integration $I$ defined in eq. 13 conserves a constant value on the reference orbit[14].

$$
\begin{aligned}
I\left(\theta_{*}, \dot{\theta}_{*}\right) & =\frac{\dot{\theta}_{*}^{2}}{2}-\exp \left(-\int_{x_{0}}^{\theta_{*}} \frac{2 \beta(\tau)}{\alpha(\tau)} d \tau\right) \\
& \left\{\frac{y_{0}^{2}}{2}-\int_{x_{0}}^{\theta_{*}} \exp \left(-\int_{s}^{\theta_{*}} \frac{2 \beta(\tau)}{\alpha(\tau)} d \tau\right) \frac{2 \gamma(s)}{\alpha(s)} d s\right\}
\end{aligned}
$$

Introducing the new coordinates $\xi=[I, y, \dot{y}]^{T}$, the system of eq.12 can be also represented by eq.14.

$$
\left\{\begin{array}{l}
\dot{I}=\frac{2 \dot{\theta}}{\alpha(\theta)}\left[g_{y}(t) y+g_{\dot{y}}(t) \dot{y}+g_{v}(t) v-\beta(\theta) I\right] \\
\ddot{y}=v
\end{array}\right.
$$

The resulted incomplete nonlinear system of eq.14 plays an important role in developing a stabilizing controller in the next section.

\section{Controller Design}

The state-space representation of the system in eq.14 is:

$$
\dot{\xi}=A(t) \xi+b(t) v
$$

where $\xi=[I, y, \dot{y}]^{T}$, and:

$$
\begin{aligned}
& A(t)=\left[\begin{array}{ccc}
-\beta(\theta) \frac{2 \dot{\theta}}{\alpha(\theta)} & g_{y}(t) \frac{2 \dot{\theta}}{\alpha(\theta)} & g_{\dot{y}}(t) \frac{2 \dot{\theta}}{\alpha(\theta)} \\
0 & 0 & 1 \\
0 & 0 & 0
\end{array}\right] \\
& b(t)=\left[g_{v}(t) \frac{2 \dot{\theta}}{\alpha(\theta)} \quad 0 \quad 1\right]^{T}
\end{aligned}
$$

One choice of the feedback controller $v$ to exponentially stabilize the linear periodic system (eq.15), can be inspired from [16] where an LQR control is applied. The feedback controller can take the following form:

$$
v=-\Gamma^{-1} b(t) R(t) \xi
$$

where $R(t)$ is a symmetric matrix $R(t)=R(t)^{T}$ for all $t \in\left[0, T_{p}\right]$, periodic $R(t)=R\left(t+T_{p}\right)$, and satisfies the Riccati equation:

$\dot{R}(t)+A(t)^{T} R(t)+R(t) A(t)+G=R(t) b(t) \Gamma^{-1} b(t)^{T} R(t)$

$\Gamma$ is a positive scaler and $G$ is a $(3 \times 3)$ positive symmetric matrix.

The obtained control diagram is shown in Fig.4. To resume, the KGS model is reduced via insertion of the VCs given in eq.5. The introduction of the full integral $I$, forms a partly linearised system for which the stabilizer (16) is designed. The stabilizer output $v$ is then transformed using eq. 11 in order to get the KGS input $u$.

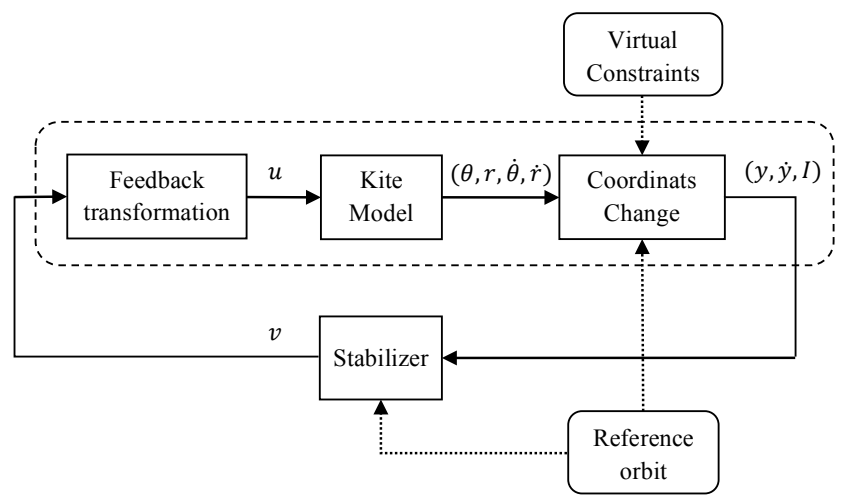

Fig. 4. Control block diagram.

To finish, the obtained solution for the reduced system of eq. 6 is a solution of the closed-loop KGS system. That is expressed by Theorem.1, that is the reformulation of (Theorem 3 - [15]) for the KGS considered case.

Theorem 1: Considering the under-actuated Kite Generator System of eq.4, which has 2 degrees of freedom, the tether's inclination and length $(\theta, r)$, and one control input, the attack angle $\alpha$, and given that:

- Applying the virtual constraint of eq.5 on the system results in a reduced system (eq.6) for which there is a periodic solution $\theta_{*}(t)$.

- $\operatorname{det} L(\theta, y) \neq 0, K(\theta, y) \neq 0 \forall(\theta, y)$

- The partial feedback linearization results in the linear periodic system of eq.14 which is completely controllable over its period.

Applying the designed control law of eq.16 on the KGS after transforming it using eq. 11 grant the chosen periodic solution $\theta_{*}(t)$ of eq.7 to be an exponentially orbitally stable for the KGS.

Proof: Since the virtual constraint is enforced using the control $v$ of eq.16 then the reduced system's solution $\theta_{*}(t)$ 
is a solution for the closed-loop KGS, and the LQR-based designed controller of eq.16 is exponentially orbitally stable according to the Proposition 4 of [15].

\section{Simulation Results}

To show the effectiveness of the proposed feedback control, a simulation study was performed using the coefficients of the experimental set-up of GIPSA-Lab [10]. They are given in Table.V.

\begin{tabular}{c|c|c} 
symbol & name & value \\
\hline$M$ & mass & $0.1 \mathrm{Kg}$ \\
$M_{I M}$ & rotor's mass & $0.0481 \mathrm{Kg}$ \\
$\rho$ & air density & $1.225 \mathrm{Kg} / \mathrm{m}^{3}$ \\
$S$ & wing area & $0.1375 \mathrm{~m}^{2}$ \\
$e$ & Oswald's factor & 0.7 \\
$\lambda$ & aspect ratio & 2.5 \\
$\partial C L / \partial \alpha$ & lift derivative w.r.t. $\alpha$ & $0.05 \mathrm{deg}-1$ \\
$C_{D 0}$ & zero lift drag & 0.07 \\
$V$ & mean air speed & $6 \mathrm{~m} / \mathrm{s}$ \\
$T$ & The tether's tension & $3 \mathrm{~N} . \mathrm{m}$ \\
\multicolumn{2}{c}{ TABLE I } \\
COEFFICIENTS FOR THE SIMULATION STUDY.
\end{tabular}

Our objective is to stabilize the system around a periodic orbit while controling the attack angle only.

Starting from an arbitrary point $(\theta, r, \dot{\theta}, \dot{r})$ within the power region of the kite, the application of proposed virtual constraints-based control developed in this paper gives the closed loop behaviour of Fig.5. One can clearly see the effectiveness of the proposed feedback control. Several initial conditions have been tested and for all of them, the trajectories have stabilized on a periodic orbit in a short time. The speed of convergence depends on the gain of the feedback control $v$.

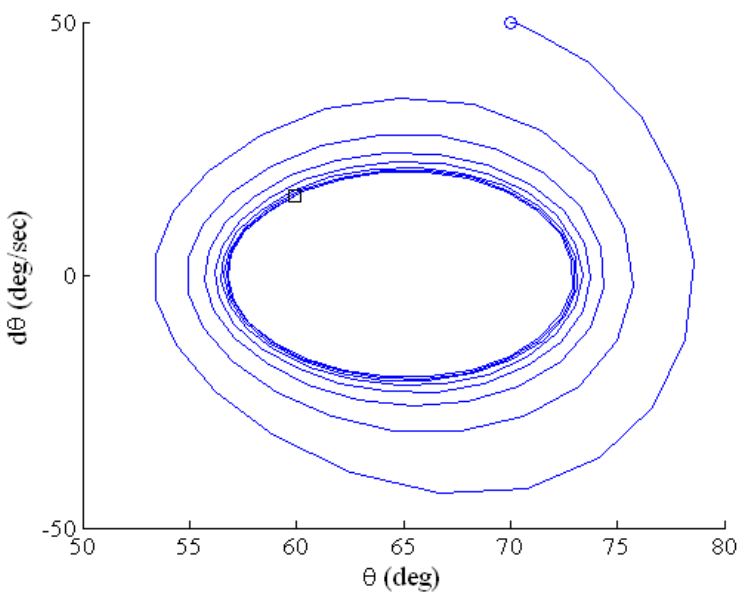

Fig. 5. The closed loop system's portrait.

In Fig.6, Fig.7 and Fig.8, the temporal evolution of the tether's length $r$ and angle $\theta$ of the KGS and the applied controls are respectively shown. Though these results may be improved through suitable choice of $\Gamma$ and $G$, one can still see the effectiveness of the proposed control for this first approach.
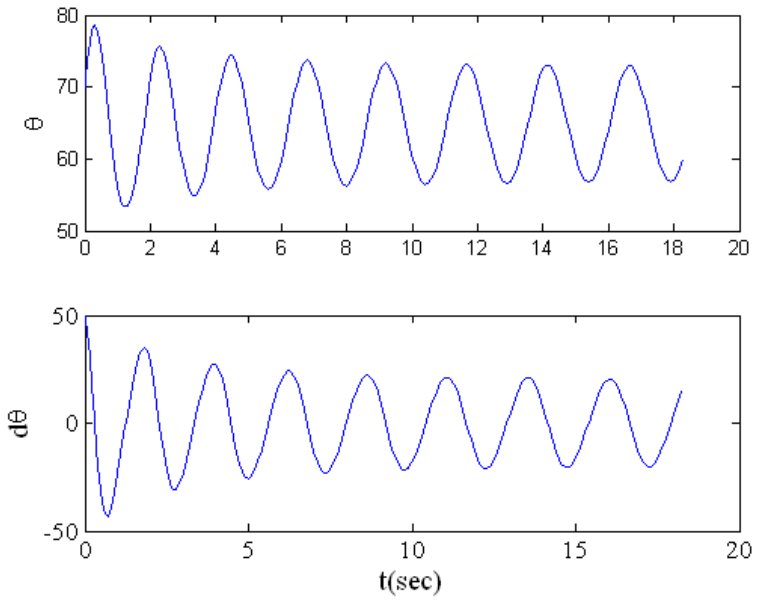

Fig. 6. The evolution of $\theta$ and $\dot{\theta}$ of the KGS.
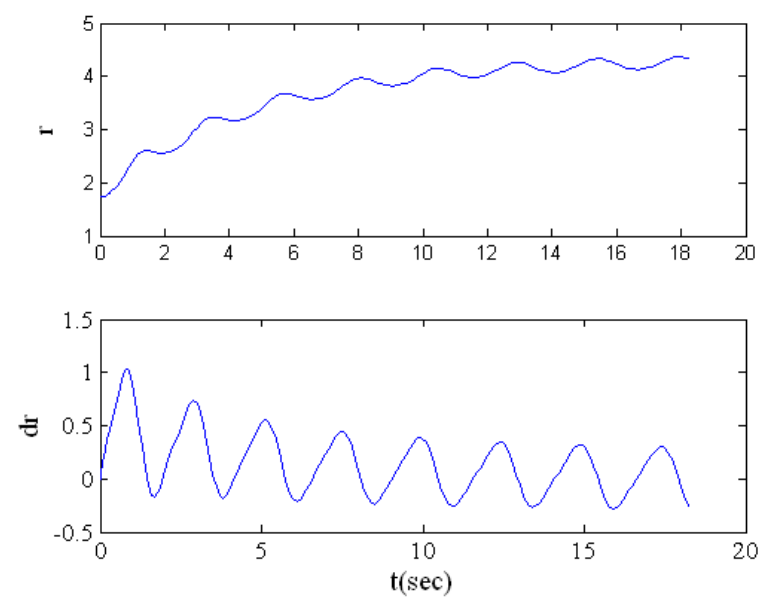

Fig. 7. The evolution of $r$ and $\dot{r}$ of the KGS.

\section{Conclusions And Perspectives}

In this paper, we have proposed the application of a state feedback control law that ensures exponential orbital stabilization of a periodic target motion for the case of a Kite generator system (KGS). The motion planning was carried out using the virtual constraint approach. Using this approach allows a fast in-loop control method that is robust against disturbances.

Numerical simulations have shown the effectiveness of the proposed control strategy and the convergence to the preplanned motion, even when initialized at an angle away from the reference orbit.

This promising result will be applied on the experimental set-up of GIPSA-Lab. It will be combined with our previous study using observer-based control [10]. In addition, more research is needed to apply the proposed control strategy on a real scale system which is constructed by GIPSA-lab. The reader can visit the following website to see the authors' 


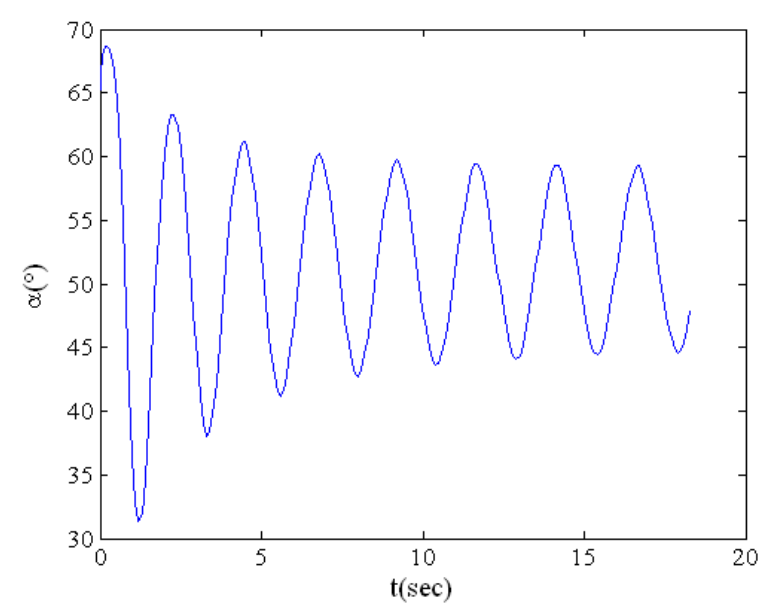

Fig. 8. The applied control for the studied KGS.

actual activities in this field ${ }^{2}$.

Finally, a special attention will be given to reference orbits that maximizes the extracted/produced energy while taking into consideration the wind variations and the bounded angle of attack.

\section{REFERENCES}

[1] M.S. Ahmed, A. Hably, and S. Bacha. Power maximization of a closed-orbit kite generator system. In CDC - 50th Conference on Decision and Control Conference, Orlando, USA, pages 7717-7722. IEEE, December 2011.

[2] M.S. Ahmed, A. Hably, and S. Bacha. High altitude wind power systems: A survey on flexible power kites. In ICEM - XXth International Conference on Electrical Machines, Marseille, France, pages 2085-2091. IEEE, September 2012.

[3] I. Argatov, P. Rautakorpi, and R. Silvennoinen. Estimation of the mechanical energy output of the kite wind generator. Renewable Energy, 34(6):1525-1532, 2009.

[4] M. Canale, L. Fagiano, and M. Milanese. High altitude wind energy generation using controlled power kites. IEEE Transactions On Control Systems Technology, 18(2):279-293, 2010.

[5] C. Canudas-de Wit. On the concept of virtual constraints as a tool for walking robot control and balancing. Annual Reviews in Control, 28(2):157-166, 2004.

[6] L. Consolini and M. Maggiore. Virtual holonomic constraints for euler-lagrange systems. In Symposium on Nonlinear Control Systems (NOLCOS), 2010.

[7] L. Fagiano, M. Milanese, and D. Piga. Optimization of airborne wind energy generators. International Journal of Robust and Nonlinear Control, 22(18):2055-2083, 2011.

[8] L. Freidovich, A. Robertsson, A. Shiriaev, and Rolf Johansson. Periodic motions of the pendubot via virtual holonomic constraints: Theory and experiments. Automatica, 44(3):785-791, 2008.

[9] A. Furey and I. Harvey. Evolution of neural networks for active control of tethered airfoils. Advances in Artificial Life, pages 746-755, 2007.

[10] A. Hably, R. Lozano, M. Alamir, and J. Dumon. Observer-based control of a tethered wing wind power system : indoor real-time experiment. In ACC - American Control Conference, Washington, DC, USA, June 2013.

[11] A. Ilzhofer, B. Houska, and M. Diehl. Nonlinear MPC of kites under varying wind conditions for a new class of large-scale wind power generators. International Journal Of Robust And Nonlinear Control, 17(17):1590-1599, 2007.

[12] M. Ippolito. Vertical axis wind turbine with control system steering kites, February 27 2008. EP Patent 1,672,214.

${ }^{2}$ http://www.gipsa-lab.grenoble-inp.fr/recherche/platesformes.php?id plateforme $=70$.
[13] A. Shiriaev and C. Canudas-de Wit. Virtual constraints: a constructive tool for orbital stabilization of under-actuated nonlinear systems. Personal Communication, 2003.

[14] A. Shiriaev, L. Freidovich, and S. Gusev. Transverse linearization for controlled mechanical systems with several passive degrees of freedom. IEEE Transactions on Automatic Control, 55(4):893-906, 2010 .

[15] A. Shiriaev, J.W. Perram, and C. Canudas-de Wit. Constructive tool for orbital stabilization of underactuated nonlinear systems: Virtual constraints approach. IEEE Transactions on Automatic Control, 50(8):1164-1176, 2005.

[16] A. Shiriaev, A. Robertsson, J. Perram, and A. Sandberg. Periodic motion planning for virtually constrained (hybrid) mechanical systems. In CDC-ECC'O5 - 44th IEEE Conference on Decision and Control, European Control Conference, Seville, Spain, pages 4035-4040. IEEE, December 2005.

[17] A Shiriaev, A Robertsson, J Perram, and A Sandberg. Periodic motion planning for virtually constrained euler-lagrange systems. Systems \& control letters, 55(11):900-907, 2006.

[18] S. Westerberg, U. Mettin, and A. Shiriaev. Motion planning and control of an underactuated 3dof helicopter. In IROS - IEEE/RSJ Intelligent Robots and Systems, Leiden, Netherlands, pages 37593764. IEEE, June 2010.

[19] P. Williams, B. Lansdorp, and W. Ockels. Modeling and control of a kite on a variable length flexible inelastic tether. In AIAA Guidance, navigation and control conference, South Carolina, USA, 2007.

\section{APPENDIX}

The detailed expression of system (4) are given by the following expressions:

- The Inertia matrix:

$$
D(\theta, r)=\left[\begin{array}{cc}
M r & 0 \\
0 & \left(M+M_{I M}\right)
\end{array}\right]
$$

- The Coriolis matrix:

$$
C(\theta, r, \dot{\theta}, \dot{r})=\left[\begin{array}{cc}
2 M \dot{r} & 0 \\
-M r \dot{\theta} & 0
\end{array}\right]
$$

- The Potential energy function:

$$
\begin{aligned}
\nabla P(\theta, r) & =\left[\begin{array}{c}
b v_{r}^{2} \sin \left(\theta-\alpha_{w}\right) \\
-b v_{r}^{2} \cos \left(\theta-i n t \alpha_{w}\right)
\end{array}\right] \\
& -\left[\begin{array}{c}
a v_{r}^{2}\left(\frac{\partial C_{l}}{\partial \alpha} \alpha_{w}+C_{L 0}\right) \cos \left(\theta-\alpha_{w}\right) \\
a v_{r}^{2}\left(\frac{\partial C_{l}}{\partial \alpha} \alpha_{w}+C_{L 0}\right) \sin \left(\theta-\alpha_{w}\right)
\end{array}\right] \\
& +\left[\begin{array}{c}
W \cos \theta \\
W \sin \theta+T
\end{array}\right]
\end{aligned}
$$

- The control matrix:

$$
B=a v_{r}^{2} \frac{\partial C_{l}}{\partial \alpha}\left[\begin{array}{c}
\cos \left(\theta-\alpha_{w}\right) \\
\sin \left(\theta-\alpha_{w}\right)
\end{array}\right]
$$

